\title{
High-Dimensional Integrator
}

DOI: $10.21105 /$ joss. 00437

\section{Software}

- Review ¿

- Repository ¿a

- Archive ca

Submitted: 11 October 2017

Published: 15 December 2017

\section{Licence}

Authors of JOSS papers retain copyright and release the work under a Creative Commons Attribution 4.0 International License (CC-BY).

\section{Ilja Honkonen ${ }^{1}$}

1 Finnish Meteorological Institute

\section{Summary}

HDIntegrator (Honkonen 2017) is a program for parallel numerical integration of functions in arbitrary number of dimensions. It is implemented in Python and parallelized using the Message Passing Interface (Forum 1995) via mpi4py (Dalcín, Paz, and Storti 2005). HDIntegrator is a wrapper program that divides the integration region into rectangular subvolumes and uses a separate serial program (the integrand) for evaluating the integral within those subvolumes. Integration of a subvolume is stopped when one of the userspecified criteria for convergence is fulfilled, otherwise the subvolume is divided into two smaller subvolumes and integration is continued. Communication between HDIntegrator and integrand is handled via standard input and output in ASCII format.

\section{Statement of need}

This program was developed due to the need for a free and open source software (FOSS) for adaptive and parallel calculation of numerical integrals. Several FOSS packages exist for numerical integration (e.g. Mark Galassi 2017, Johnson (2017)) but none seem to support parallel integration in arbitrary number of dimensions using a supercomputer. HDIntegrator is implemented as a parallel wrapper program that can take advantage of existing serial software for numerical integration. This also allows one to easily develop and use efficient integrands separately from the parallel wrapper, that use specialized hardware or environments such as GPUs and even cloud computing. This program is currently used for studying turbulence via numerical integration of functional integrals.

\section{References}

Dalcín, Lisandro, Rodrigo Paz, and Mario Storti. 2005. "MPI for Python." Journal of Parallel and Distributed Computing 65 (9):1108-15. https://doi.org/10.1016/j.jpdc.2005. 03.010.

Forum, Message Passing Interface. 1995. "MPI: A Message-Passing Interface Standard, Version 1.1." 1995. http://mpi-forum.org/docs/mpi-1.1/mpi-11-html/mpi-report.html.

Honkonen, Ilja. 2017. "HDIntegrator." 2017. https://github.com/iljah/hdintegrator. Johnson, Steven G. 2017. "Cubature." 2017. https://github.com/stevengj/cubature.

Mark Galassi, et al., James Theiler. 2017. "GSL - Gnu Scientific Library, Version 2.4." 2017. https://www.gnu.org/software/gsl/. 